\title{
Correlating the Structure and Composition of 2D Materials with Their Catalytic Activity
}

\author{
Oren Meiron ${ }^{1,2}$, Vasu Kuraganti ${ }^{1,2}$, Lothar Houben ${ }^{3}$ and Maya Bar Sadan ${ }^{1,2}$ \\ 1. Chemistry Department, Ben Gurion University, Israel \\ 2. Ilse Katz Institute for Nanoscale Science \& Technology, Ben Gurion University, Israel \\ 3. Department of Chemical Research Support, Weizmann Institute of Science, Israel.
}

Nanostructures are one of the most extensively researched systems in nanoscience. Various materials are investigated due to their size dependent optical properties or due to their enhanced functionality as catalysts. Although they have been extensively researched for a few decades now, even today new fabrication routes are still explored to improve properties and to gain precise control of their structure. While reports on the optical properties of single particles are available, the quantitative characterization of atomic order on a single particle level and the growth mechanism that resulted in that specific rearrangement, are still generally missing. The majority of characterization procedures are performed on ensembles that average properties and may hinder the understanding of fundamental aspects in the colloidal synthesis.

Atomic resolution analysis, which has emerged with aberration corrected instruments, has mainly provided analysis of few particles per sample. It is now, due to the Cc correction that offers superior resolutions in low voltages that the atomic ordering can be achieved on a routine basis to deliver new statistical data. New hardware facilitates efficient acquisition of chemical data by EDS without causing deformation due to beam damage. We use these state-of-the-art instrumentation to understand growth processes and to correlate the atomic structure with properties.

Here, the formation of ternary compounds of transition metal di-chalcogenides (TMDs) by wet chemistry will be described. Specifically, the doping of TMDs with other transition metals and the impact it has on their catalytic properties for hydrogen production will be presented.

The growth mechanism of nanoflowers nanostructures of TMDs was revealed using electron tomography [1]. Using tomography of the nanostructures at different times of the reactions, we showed that the growth mechanism goes through the formations of amorphous pockets, where each crystalizes into a sponge-like structure in the shape of a nanoflower. This growth mechanism allows for facile doping of the materials by adding the dopants either at the beginning or at the end of the reaction, thus forming a homogenous material or a graded one. We have used this approach to dope $\mathrm{MoS}_{2}$ with $\mathrm{Ru}$, and significantly improving its catalytic activity towards hydrogen production. In addition, we prepared many ternary compounds, and correlated the composition with catalytic activity.

In addition, we have surveyed the structural defects within the nanoflowers in order to correlate them with the catalytic activity. Typical defect motifs will be shown and discussed. This work aims at correlating the atomic-scale structures with the catalytic activity, and for that goal to be achieved, there is a need to understand the dopant sites and the atomic scale arrangement within the $\mathrm{MoS}_{2}$ lattice. The use of high resolution electron microscopy with other characterization methods allows first the understanding of the structural features of the materials and thereafter it will serve to understand the origin of catalytic activity.

[1] O.E. Meiron, L. Houben, and M. Bar-Sadan, RSC Adv., 5 (2015) p. 88108-88114 


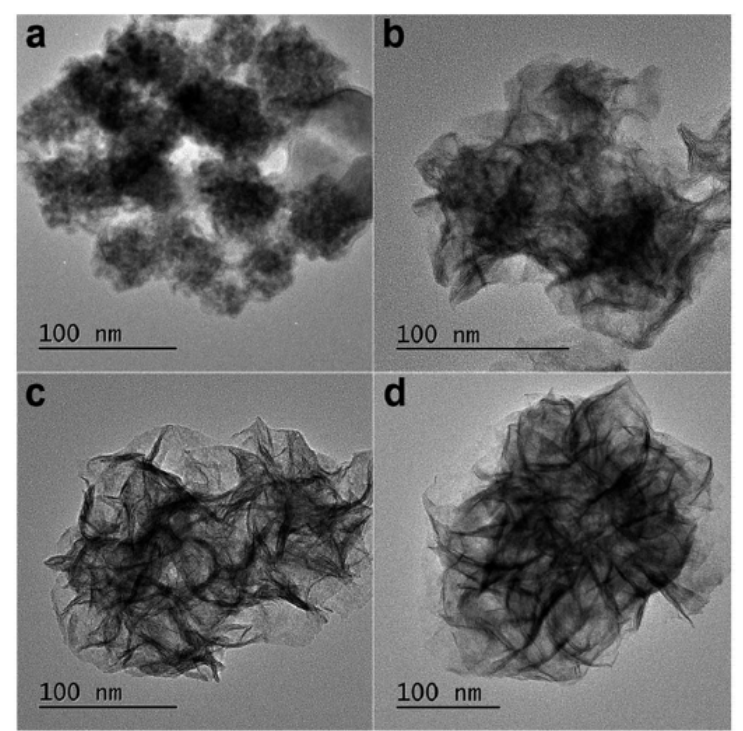

Figure 1. Time series analysis of $\mathrm{MoSe}_{2}$ particles. TEM images of MoSe 2 nanoflowers taken at times: (a) 1 min. (b) 3 min. (c) 5 min. (d) 10 min.

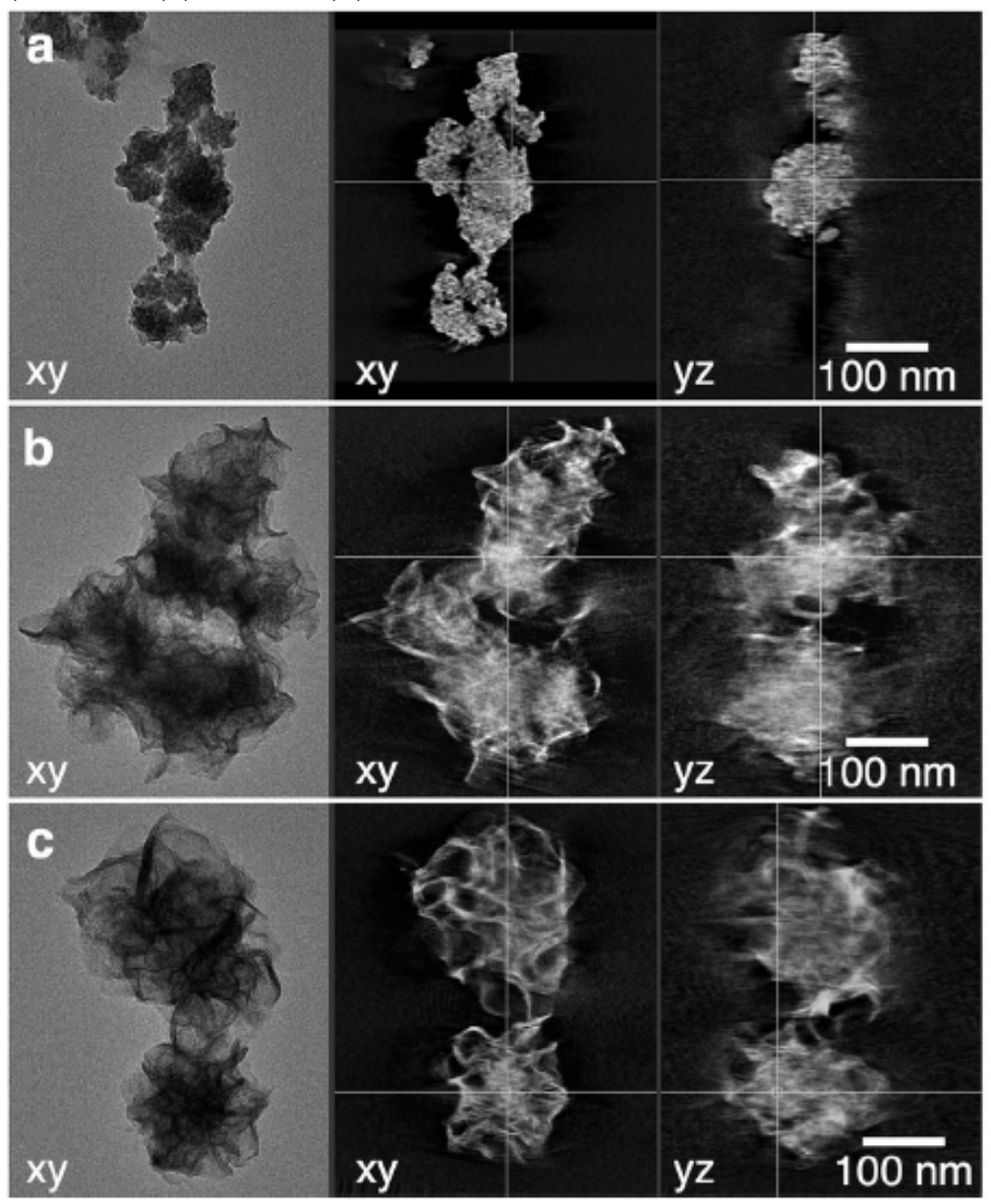

Figure 2. Tomogram data for the time series analysis of $\mathrm{MoSe}_{2}$ particles. TEM images and tomogram slices of $\mathrm{MoSe}_{2}$ nanoflowers taken at times: (a) 1 min. (b) 3 min. (c) 5 min. Each panel displays a zero tilt TEM image in the xy-projection plane on the left, and two central tomogram slices in the xy- and yz-plane on the right. 